\title{
Retinal microvasculature alterations in myopic anisometropia on optical coherence tomography angiography
}

Xiaorong Li ( $\nabla$ xiaorli@163.com)

Tianjin Medical University Eye Hospital https://orcid.org/0000-0003-0641-2797

liangzhang Tan

Tianjin Medical University Eye Hospital

lu Chen

Tianjin Medical University Eye Hospital

shaochong Bu

Tianjin Medical University Eye Hospital

yuanfeng jiang

Tianjin Medical University Eye Hospital

zhiqiong $\mathrm{Ma}$

Zhejiang Xiaoshan Hospital

xue Gong

Tianjin Medical University Eye Hospital

fang Tian

Tianjin Medical University Eye Hospital

hong zhang

Tianjin Medical University Eye Hospital

\section{Research article}

Keywords: myopic anisometropia, optical coherence tomography angiography, vessel density, axial length

Posted Date: February 27th, 2020

DOl: https://doi.org/10.21203/rs.2.24715/v1

License: (c) (i) This work is licensed under a Creative Commons Attribution 4.0 International License.

Read Full License 


\section{Abstract}

Background: In myopic anisometropia, investigation of alterations in the retinal capillary network may reveal the myopic pathophysiology and facilitate development of improved treatment and prevention strategies. This study aimed to evaluate alterations in retinal vessel density in patients with myopic anisometropia using optical coherence tomography angiography.

Methods: Twenty-one patients with myopic anisometropia were divided into longer axial eye and contralateral eye groups, based on their axial length measurement by an IOL Master system. Automated macular and peripapillary maps and vessel density calculations were evaluated using optical coherence tomography angiography, and the findings were compared between groups.

Results: The longer axial eye group had higher superficial macular vessel density $(47.06 \pm 3.03 \% \mathrm{vs}$. $43.71 \pm 3.20 \%$, respectively; $P<0.001)$ and lower radial peripapillary capillary density $(50.11 \pm 3.56 \%$ vs. $52.34 \pm 2.74 \%$, respectively; $P=0.013)$ than did the contralateral eye group. The spherical equivalent negatively correlated with the superficial macular vessel density $(P=0.021)$ and superficial parafoveal vessel density $(P=0.014)$. No significant differences were detected in the deep macular vessel density $(49.12 \pm 6.32 \%$ vs. $46.73 \pm 5.30 \%$, respectively; $P>0.05)$, choriocapillary perfusion area $(1.92 \pm 0.18$ $\mathrm{mm} 2$ vs. $1.90 \pm 0.19 \mathrm{~mm} 2$, respectively; $P>0.05)$, or foveal avascular zone area $(0.27 \pm 0.07 \mathrm{~mm} 2$ vs. $0.28 \pm 0.07 \mathrm{~mm} 2$, respectively; $P>0.05$ ) between groups.

Conclusion: The superficial macular vessel density and radial peripapillary capillary density could be used as indicators to monitor myopic anisometropia development during early-stage retinopathy.

\section{Background}

Myopia is the leading cause of distance vision impairment worldwide, and is characterised by elongation of the eye and structural changes to the retina and choroid [1]. Myopia is associated with an increased risk of blindness ${ }^{[2]}$ and predominantly affects the young population in Asia [3]. Despite refractive correction, myopic patients have a higher risk of developing a range of vision-threatening conditions, including myopic macular degeneration, chorioretinal atrophy, and retinal detachment $[4,5]$. The complications of myopia are closely correlated with the morphological alterations of the retinal vessels [5].

Myopic anisometropia is an asymmetric phenomenon of ametropia between the two eyes of an individual with a difference of $>1.00 \mathrm{D}$ in the spherical equivalent (SE), which is typically accompanied by a significant difference in axial lengths (ALs). Based on the estimation of Saw et al. [6], anisometropia affects $5-10 \%$ of the population aged $>20$ years. A number of researchers have investigated the interocular symmetry of retinal thickness in amblyopic anisometropia [7] and revealed that regional changes in retinal thickness can be used to monitor myopic development at an early stage [8]. The retinal capillary network plays a crucial role in providing nutrients and oxygen to the retina, which may be more sensitive to myopia-related changes. Therefore, in myopic anisometropia, investigating the alterations in 
the retinal capillary network may reveal the potential myopic pathophysiology and may aid the development of improved treatment and prevention strategies. However, the technical difficulties involved in imaging the retinal vasculature hinder its utility as an early indicator of the onset of retinopathy [9].

After the US Food and Drug Administration (Rockville, MD) approved the clinical use of optical coherence tomography angiography (OCTA), it has become a rapidly evolving technology that can reveal the motion of erythrocytes using an algorithm, called split spectrum amplitude decorrelation angiography [10]. It has the advantage of providing detailed information regarding the ocular vasculature. Therefore, OCTA has been applied for the assessment of retinal and choroidal diseases such as glaucoma, diabetic retinopathy, and central serous chorioretinopathy [11]. The purpose of this research was to observe the retinal vessel density alterations in people with myopia with an interocular difference of $\geq 1.50 \mathrm{D}$ and to explore potentially novel insights into the mechanisms underlying refractive error development in myopic anisometropia.

\section{Participants And Methods Study population}

Twenty-one myopic anisometropic patients were consecutively enrolled in this cross-sectional study between September 2018 and August 2019. The ethics committee of Tianjin Medical University approved the study, which was conducted in accordance with the Declaration of Helsinki. Written informed consent was obtained from each participant.

Myopia of $>0.50 \mathrm{D}$ in both eyes and an interocular difference of $\geq 1.5 \mathrm{D}$ in the SE was accepted as the threshold for myopic anisometropia. The refraction data were converted to SEs (SE = spherical component $+1 / 2$ cylindrical component). Each participant underwent a thorough ophthalmic examination including best-corrected visual acuity, intraocular pressure by Goldmann applanation tonometry, refraction by automatic refractometer, slit-lamp examination, fundoscopy, and AL measurement by an IOL Master system (Carl Zeiss, Meditec, Germany). The participants were divided into the longer axial eye group (LAG) and contralateral eye group (CLG), based on their AL measurement.

The exclusion criteria were as follows: myopia of >6.00 D; amblyopia; ocular hypertension; a history of systemic diseases such as hypertension, diabetes mellitus, and cardiovascular disease, which might interfere with ocular circulation; ocular surgery; and other evidence of retinal pathology such as retinal vascular diseases.

\section{Image acquisition}

The OCTA images were obtained using RTVue XR OCT (Optovue, Inc., Fremont, CA, USA; Software V.2017.1.0.155). A split-spectrum amplitude decorrelation algorithm was used to extract the OCTA images, with A-scans of $70,000 \mathrm{HZ}$ scans per s. The $3 \times 3 \mathrm{~mm}$ angio-retina mode was centred on the fovea, and the $4.5 \times 4.5 \mathrm{~mm}$ angio-disc mode was centred on the optic disc. The captured images 
contained two repeated volumes of $304 \mathrm{~B}$-scans. During image processing, the Motion Correction Technology function was used to correct the horizontal and vertical scans to offset eye movement [12]. The viewing software performed automatic segregation to generate images. The following parameters were evaluated: superficial macular vessel density, deep macular vessel density, choriocapillary perfusion (CCP) area, foveal avascular zone (FAZ) area, and radial peripapillary capillary (RPC) density.

All scans with layer segmentation errors, signal strength indices of $<50$, or significant motion artifacts were excluded.

\section{Image selection and analysis}

Macular angiogram

A $3 \times 3 \mathrm{~mm}$ macular angiogram of the superficial and deep layers was analysed using Optovue software with density function. The FAZ area was automatically captured using the non-flow function at the level of the corresponding vascular networks [13]. The boundaries of the superficial retinal layer extended from $3 \mu \mathrm{m}$ below the inner limiting membrane (ILM) to $15 \mu \mathrm{m}$ below the inner plexiform layer (IPL), and the boundaries of the deep retinal layer extended from $15 \mu \mathrm{m}$ to $70 \mu \mathrm{m}$ below the IPL [14]. Vessel density was separately processed in four regions (superior-hemi, inferior-hemi, fovea, and parafovea). Blood flow was calculated automatically in a selected area of $3,144 \mathrm{~mm}^{2}$ centred on the fovea in the choriocapillary from $31 \mu \mathrm{m}$ to $59 \mu \mathrm{m}$ below the retinal pigment epithelium [13].

Optic disc angiogram

A $4.5 \times 4.5 \mathrm{~mm}$ optic disc angiogram was analysed using Optovue software with density function. The average vessel density was automatically calculated from the nerve head segment, which extended from the ILM to $150 \mu \mathrm{m}$ below the ILM. The average RPC density was automatically calculated in a $0.75-\mathrm{mm}$ wide elliptical annulus region, which extended from the optic disc boundary. The RPC density was separately processed in six regions (superior-hemi, inferior-hemi, temporal, superior, nasal, and inferior) [13].

\section{Statistical analysis}

Statistical analyses were performed using SPSS version 19.0 (SPSS, Inc., Chicago, IL, USA). Data are presented as the mean \pm standard deviation. Paired t-tests were used to assess the interocular differences for measurements obtained from the OCTA. Pearson's correlation and linear regression analysis were used to determine the relationships between the AL and SE and vessel density. A P-value of $<0.05$ was considered statistically significant.

\section{Results}

\section{Demographics}


A total of 21 individuals ( 10 men and 11 women) were included in this study. The mean age was $27.67 \pm$ 6.40 years (range $20-59$ years). The AL in the LAG and the CLG was $25.78 \pm 0.91 \mathrm{~mm}$ and $24.28 \pm$ $0.93 \mathrm{~mm}$, respectively $(P<0.001)$. The SE in the $L A G$ and $C L G$ was $-4.81 \pm 1.30 \mathrm{D}$ and $-2.11 \pm 1.40 \mathrm{D}$, respectively $(P<0.001)$. The demographics of the enrolled participants are provided in Tables 1 and 2.

Table 1

Participant demographics

\begin{tabular}{ll} 
Variables & Mean \pm SD \\
\hline Sex, male/female $(\mathrm{n})$ & $10 / 11$ \\
Age (years) & $27.67 \pm 6.40$ \\
SBP $(\mathrm{mmHg})$ & $117.62 \pm 7.31$ \\
DBP $(\mathrm{mmHg})$ & $74.67 \pm 8.10$ \\
MAP $(\mathrm{mmHg})$ & $88.98 \pm 6.35$ \\
Pulse $(\mathrm{bpm})$ & $70.52 \pm 6.23$
\end{tabular}

MAP = DBP + 1/3 (SBP - DBP). bpm: beats per min. DBP: diastolic blood pressure. SBP: systolic blood pressure. MAP: mean arterial pressure.

Table 2

Participant demographics according to AL

\begin{tabular}{|llll|}
\hline Variables & LAG & CLG & P \\
\hline BCVA (logMAR) & $-0.02 \pm 0.04$ & $-0.02 \pm 0.04$ & 0.576 \\
\hline SE $(\mathrm{D})$ & $-4.81 \pm 1.30$ & $-2.11 \pm 1.40$ & $<0.001$ \\
\hline IOP $(\mathrm{mmHg})$ & $16.46 \pm 3.08$ & $16.79 \pm 2.77$ & 0.324 \\
$\mathrm{CC} T(\mu \mathrm{m})$ & $533.57 \pm 34.72$ & $534.62 \pm 35.16$ & 0.415 \\
\hline $\mathrm{ACD}(\mathrm{mm})$ & $3.13 \pm 0.16$ & $3.13 \pm 0.17$ & 0.803 \\
\hline $\mathrm{AL}(\mathrm{mm})$ & $25.78 \pm 0.91$ & $24.28 \pm 0.93$ & $<0.001$ \\
\hline MOPP $(\mathrm{mmHg})$ & $48.35 \pm 3.89$ & $47.91 \pm 4.20$ & 0.180 \\
\hline $\begin{array}{l}\text { MOPP = 2/3(MAP - IOP). ACD: anterior chamber depth. AL: axial length. BCVA: best corrected visual } \\
\text { acuity. CCT: central corneal thickness. CLG: contralateral eye group. IOP: intraocular pressure. LAG: } \\
\text { longer axial eye group. LogMAR: logarithm of the minimum angle of resolution. MOPP: mean ocular } \\
\text { perfusion pressure. SE: spherical equivalent. }\end{array}$ & \\
\hline
\end{tabular}

\section{Macular vessel density}

In the superficial layers, significant differences were found in the vessel densities of all regions, with the exception of the fovea, between the LAG and CLG. The LAG had a higher superficial macular vessel 
density $(47.06 \pm 3.03 \%$ vs. $43.71 \pm 3.20 \%$, respectively; $P<0.001)$ and parafoveal vessel density $(49.55 \pm$ $3.86 \%$ vs. $46.02 \pm 3.40 \%$, respectively; $P<0.001$ ) than did the CLG.

In the deep macular layers, no significant differences were found in the vessel densities of any regions between the groups (all $P>0.05$ ). The CCP areas in the LAG and CLG were $1.92 \pm 0.18 \mathrm{~mm}^{2}$ and $1.90 \pm$ $0.19 \mathrm{~mm}^{2}$, respectively $(P>0.05)$. The FAZ areas in the LAG and CLG were $0.27 \pm 0.07 \mathrm{~mm}^{2}$ and $0.28 \pm$ $0.07 \mathrm{~mm}^{2}$, respectively $(P>0.05)$ (Table 3$)$.

Table 3

Macular vessel density

\begin{tabular}{|llll|}
\hline Variables & LAG & CLG & P \\
\hline Superficial macular vessel density (\%) & $47.06 \pm 3.03$ & $43.71 \pm 3.20$ & $<0.001$ \\
\hline Superficial superior-hemi vessel density (\%) & $47.20 \pm 3.29$ & $43.74 \pm 2.74$ & $<0.001$ \\
\hline Superficial inferior-hemi vessel density (\%) & $46.91 \pm 2.95$ & $43.70 \pm 3.96$ & $<0.001$ \\
\hline Superficial foveal vessel density (\%) & $17.44 \pm 4.72$ & $15.88 \pm 4.84$ & 0.051 \\
\hline Superficial parafoveal vessel density (\%) & $49.55 \pm 3.86$ & $46.02 \pm 3.40$ & $<0.001$ \\
\hline Deep macular vessel density (\%) & $49.12 \pm 6.32$ & $46.73 \pm 5.30$ & 0.134 \\
\hline Deep superior-hemi vessel density (\%) & $49.26 \pm 6.35$ & $47.18 \pm 5.55$ & 0.187 \\
\hline Deep inferior-hemi vessel density (\%) & $48.96 \pm 6.33$ & $46.28 \pm 5.20$ & 0.105 \\
\hline Deep foveal vessel density (\%) & $32.30 \pm 8.59$ & $32.08 \pm 6.92$ & 0.836 \\
\hline Deep parafoveal vessel density (\%) & $52.07 \pm 6.05$ & $49.30 \pm 5.86$ & 0.094 \\
\hline CCP area $\left(m^{2}\right.$ ) & $1.92 \pm 0.18$ & $1.90 \pm 0.19$ & 0.541 \\
\hline FAZ area (mm ${ }^{2}$ ) & $0.27 \pm 0.07$ & $0.28 \pm 0.07$ & 0.298 \\
\hline FAZ: foveal avascular zone. CCP: choriocapillary perfusion. & & \\
\hline
\end{tabular}

\section{RPC density}

The LAG presented with a significant decrease in the RPC density compared to that in the CLG $(50.11 \pm$ $3.56 \%$ vs. $52.34 \pm 2.74 \%$, respectively, $P=0.013$ ). Further multiple comparisons revealed significant differences in the superior-hemi, inferior-hemi, temporal, superior, and inferior regions, but not in the nasal region in both groups (Table 4). 
Table 4

RPC density

\begin{tabular}{|llll|}
\hline Variables & LAG & CLG & P \\
\hline Optic disc RPC density (\%) & $50.11 \pm 3.56$ & $52.34 \pm 2.74$ & 0.013 \\
\hline Optic disc superior-hemi vessel density (\%) & $50.79 \pm 3.00$ & $52.30 \pm 2.69$ & 0.029 \\
\hline Optic disc inferior-hemi vessel density (\%) & $49.37 \pm 4.51$ & $52.35 \pm 3.26$ & 0.018 \\
\hline Optic disc temporal vessel density (\%) & $54.33 \pm 3.61$ & $56.24 \pm 4.88$ & 0.049 \\
\hline Optic disc superior vessel density (\%) & $50.71 \pm 4.50$ & $52.90 \pm 3.53$ & 0.042 \\
\hline Optic disc nasal vessel density (\%) & $45.52 \pm 4.16$ & $47.43 \pm 3.78$ & 0.052 \\
\hline Optic disc inferior vessel density (\%) & $50.48 \pm 6.27$ & $53.81 \pm 4.17$ & 0.026 \\
\hline RPC: radial peripapillary capillary. & & & \\
\hline
\end{tabular}

\section{Correlation between AL and SE and vessel density}

In the superficial macular layer, the SE negatively correlated with the macular vessel density $(P=0.021)$ and parafoveal vessel density $(P=0.014)$ (Fig. 1). No significant correlation was found between the SE and deep macular vessel density, deep parafoveal vessel density, foveal vessel density, or RPC density (all $P>0.05)$. No significant correlation was found between the AL and the macular vessel density, foveal vessel density, parafoveal vessel density, or RPC density (all P >0.05).

\section{Discussion}

The study aimed to evaluate the retinal vessel density to explore potentially novel insights into the mechanisms underlying refractive error development in patients with myopic anisometropia using optical coherence tomography angiography. The anterior segment parameters of patients with myopic anisometropia were highly symmetrical in both groups (Table 1). This is in accordance with previous research, which found that asymmetric axial elongation of the vitreous cavity was the leading cause of myopic anisometropia [15].

This study demonstrated that the vessel density in the superficial macular layer in the LAG was significantly increased in all regions, with the exception of the foveal region, compared to that in the CLG. Yang et al. [16] demonstrated that varying degrees of myopia did not affect macular vessel density in healthy young adults. However, Al-Sheikh et al. [17] concluded that severe myopia was associated with decreased macular vessel density, in accordance with another previous study [18]. The superficial macular vascular plexus primarily supplies blood to the IPL, ganglion cell layer, and retinal nerve fibre layer (RNFL), and is not uniformly distributed from the margin of the FAZ to the parafoveal region [19]. Therefore, we believe that a compensatory increase in the oxygen and nutrient demands of the superficial macular vessel density occurs to ensure normal visual function, accompanied by axial elongation. We 
also noticed that the fovea is primarily an avascular region; therefore, it is less sensitive to certain differences in foveal vessel density.

Moreover, the RPC density was significantly decreased in the LAG compared to the CLG. The RPC is primarily responsible for supplying the nutritional requirements of the retinal ganglion cells. Previous studies have noted that the RPC density was remarkably increased in the capillary networks of the arcuate fibre region, which may play a crucial role in neuronal function [20]. Shimada et al. [21] reported that individuals with severe myopia had reduced peripapillary retinal perfusion compared to that in emmetropic eyes. In the present study, the decrease in the RPC density could have been caused by the loss of the RNFL in the corresponding area, which may have affected the demand for regional oxygen or vascular supply in the peripapillary area, thereby triggering the retinal vascular adjustment via autoregulatory mechanisms [22]. With the progression of myopia in the longer axial eyes, the eyeball is being elongated and stretched, resulting in decreased blood flow. However, the blood flow was not significantly decreased in the nasal region in the current study. This could have been associated with peripapillary atrophy, which often occurs from the side of the optic disk. Further research with a larger sample size is needed to clarify the exact underlying mechanism.

We did not find any changes in the deep macular vessel density in any regions. The deep macular plexus regions receive a portion of nutrients from the choroidal blood flow, which was not sufficiently sensitive to perceive the differences. There were no significant differences in the FAZ area measurements between groups. Indeed, the FAZ area was found to vary considerably among healthy participants based on age and other factors [23]. Our study also found no significant difference in the CCP area. Although Mo et al. observed decreased vessel density in myopic compared to emmetropic eyes [24], the chorioretinal atrophy areas may have confounded the results because of the shadowing effect caused by the lesions in their study $\left[{ }^{13}\right]$. We specifically eliminated pathological retinal changes and eyes with myopia of $>6.00 \mathrm{D}$ in our research.

Furthermore, we noted that the SE negatively correlated with the superficial macular vessel density and superficial parafoveal vessel density, but was not associated with the deep macular vessel density or RPC density. Our study did not identify any association between the AL and the macular vessel density or the RPC density. In contrast, Spina et al. [25] reported that the ocular blood flow appeared to be negatively related to the AL. Yang et al. $\left.{ }^{16}\right]$ demonstrated that the SE did not affect the macular vessel density in young patients $(26.0 \pm 1.7$ years) with myopia without pathological retinal changes. We speculated that the refractive error alone might imply a diverse mechanism in the alteration of retinal vessel density.

The potential limitations of our study should be considered. First, we focused on non-amblyopic myopic anisometropia eyes of patients aged 20-59 years and excluded the eyes with myopia of $>6.00 \mathrm{D}$ and myopia-related retinopathies. Further longitudinal studies may help to address the question of whether retinal vessel density is altered with age. Second, even subtle ocular movements during imaging can cause significant artifacts that may confound the analysis of the results; appropriate interpretation is needed to acquire a high-quality retinal vascular network image [26]. Thirdly, the sample size was 
relatively small. Thus, further long-term studies with a greater age spectrum and larger samples might reveal greater detail regarding the changes in vessel density in myopic anisometropia.

\section{Conclusions}

Our results indicate that the longer axial eyes had higher superficial macular vessel density and lower radial peripapillary capillary density than did the contralateral eyes in patients with myopic anisometropia. Thus, the superficial macular vessel density and the radial peripapillary capillary density could be used to monitor myopic anisometropia development as an indicator at the early stages of retinopathy.

\section{Declarations}

\section{Data Availability}

The data used to support the findings of this study are available from the corresponding author on request.

\section{Conflicts of Interest}

The authors declare that there is no conflict of interest regarding the publication of this article.

\section{Acknowledgments}

This research did not receive any specific grant from funding agencies in the public, commercial, or notfor-profit sectors.

\section{References}

1. Curtin BJ, Karlin DB. Axial length measurements and fundus changes of the myopic eye. Am J Ophthalmol. 1971; 71(1): 42-53.

2. Williams KM, et al. Age of myopia onset in a British population-based twin cohort. Ophthalmic Physiol Opt. 2013; 33(3): 339-345.

3. Fricke TR, et al. Global prevalence of visual impairment associated with myopic macular degeneration and temporal trends from 2000 through 2050: systematic review, meta-analysis and modelling. Br J Ophthalmol. 2018; 102(7): 855-862.

4. Verhoeven VJ, et al. Visual consequences of refractive errors in the general population. Ophthalmology. 2015; 122(1): 101-109.

5. Kim YM, Yoon JU, Koh HJ. The analysis of lacquer crack in the assessment of myopic choroidal neovascularization. Eye. 2011; 25(7): 937-946.

6. Saw SM, et al. Prevalence rates of refractive errors in Sumatra, Indonesia. Invest Ophthalmol Vis Sci. 2002; 43(10): 3174-3180. 
7. Vincent SJ, et al. Retinal and choroidal thickness in myopic anisometropia. Invest Ophthalmo/ Vis Sci. 2013; 54(4): 2445-2456.

8. Xie R, et al. Correlation between myopia and major biometric parameters of the eye: a retrospective clinical study. Optom Vis Sci. 2009; 86(5): 503-508.

9. Li M, et al. Retinal microvascular network and microcirculation assessments in high myopia. Am J Ophthalmol. 2017; 174:56-67

10. Spaide RF, Klancnik JM Jr, Cooney MJ. Retinal vascular layers imaged by fluorescein angiography and optical coherence tomography angiography. JAMA Ophthalmol. 2015; 133(1): 45-50.

11. Tan ACS, et al. An overview of the clinical applications of optical coherence tomography angiography. Eye. 2018; 32(2): 262-286.

12. Kraus MF, et al. Quantitative 3D-OCT motion correction with tilt and illumination correction, robust similarity measure and regularization. Biomed Opt Express. 2014; 5(8): 2591-2613.

13. Milani $P$, et al. Vessel density, retinal thickness, and choriocapillaris vascular flow in myopic eyes on OCT angiography. Graefes Arch Clin Exp Ophthalmol. 2018; 256(8): 1419-1427.

14. Agemy SA, et al. Retinal vascular perfusion density mapping using optical coherence tomography angiography in normals and diabetic retinopathy patients. Retina. 2015; 35(11): 2353-2363.

15. Logan NS, et al. Posterior retinal contour in adult human anisomyopia. Invest Ophthalmol Vis Sci. 2004; 45(7): 2152-2162.

16. Yang S, et al. Quantification of macular vascular density using optical coherence tomography angiography and its relationship with retinal thickness in myopic eyes of young adults. $J$ Ophthalmol. 2017; 1155:1-10.

17. Al-Sheikh $\mathrm{M}$, et al. Quantitative OCT angiography of the retinal microvasculature and the choriocapillaris in myopic eyes. Invest Ophthalmol Vis Sci. 2017; 58(4): 2063-2069.

18. $\mathrm{Li} \mathrm{H}$, et al. Lens opacity and refractive influences on the measurement of retinal vascular fractal dimension. Acta Ophthalmol. 2010; 88(6): 234-240.

19. Jiang $\mathrm{H}$, et al. In vivo characterization of retinal microvascular network in multiple sclerosis. Ophthalmology. 2016; 123(2): 437-438.

20. Tan PE, et al. Quantitative confocal imaging of the retinal microvasculature in the human retina. Invest Ophthalmol Vis Sci. 2012; 53(9): 5728-5736.

21. Shimada N, et al. Reduction of retinal blood flow in high myopia. Graefes Arch Clin Exp Ophthalmol. 2004; 242(4): 284-288.

22. Sung MS, et al. Clinical features of superficial and deep peripapillary microvascular density in healthy myopic eyes. PLoS One. 2017; 12(10): e0187160.

23. Tan CS, et al. Optical coherence tomography angiography evaluation of the parafoveal vasculature and its relationship with ocular factors. Invest Ophthalmol Vis Sci. 2016; 57(9): 224-234.

24. Mo J, et al. Vascular flow density in pathological myopia: an optical coherence tomography angiography study. BMJ Open. 2017; 7(2): 013571. 
25. Spina C L, et al. Static characteristics and dynamic functionality of retinal vessels in longer eyes with or without pathologic myopia. Graefes Arch Clin Exp Ophthalmol. 2016; 254(5): 827-834.

26. Enders $C$, et al. Quantity and quality of image artifacts in optical coherence tomography angiography. PLoS One. 2019; 14(1): e0210505.

\section{Figures}
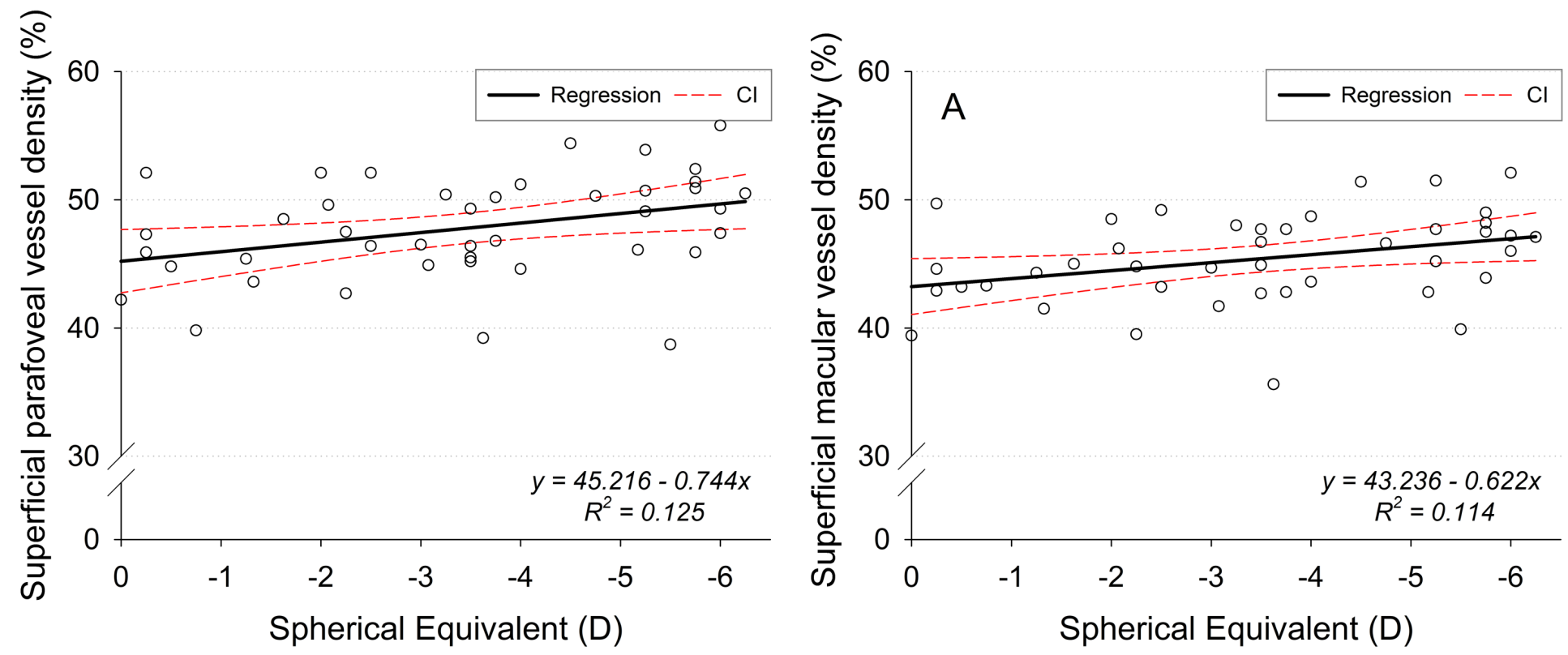

\section{Figure 1}

Scatterplots showing correlations between SE and superficial parafoveal vessel density and superficial macular vessel density. The SE negatively correlated with the superficial parafoveal vessel density (Left) and superficial macular vessel density (Right). 